\title{
Modular invariant models of lepton masses at levels 4 and 5
}

\author{
Juan Carlos Criado, ${ }^{a}$ Ferruccio Feruglio ${ }^{b}$ and Simon J.D. King ${ }^{b, c}$ \\ ${ }^{a}$ CAFPE and Departamento de Física Teórica y del Cosmos, Universidad de Granada, \\ Campus de Fuentenueva, E-18071, Granada, Spain \\ ${ }^{b}$ Dipartimento di Fisica e Astronomia 'G. Galilei', \\ Università di Padova and INFN - Sezione di Padova, \\ Via Marzolo 8, I-35131 Padua, Italy \\ ${ }^{c}$ School of Physics and Astronomy, University of Southampton, \\ Southampton, SO17 1BJ, U.K. \\ E-mail: jccriadoalamo@ugr.es, ferruccio.feruglio@pd.infn.it, \\ sjd.king@soton.ac.uk
}

ABSTRACT: We explore alternative descriptions of the charged lepton sector in modular invariant models of lepton masses and mixing angles. In addition to the modulus, the symmetry breaking sector of our models includes ordinary flavons. Neutrino mass terms depend only on the modulus and are tailored to minimize the number of free parameters. The charged lepton Yukawa couplings rely upon the flavons alone. We build modular invariant models at levels 4 and 5 , where neutrino masses are described both in terms of the Weinberg operator or through a type I seesaw mechanism. At level 4, our models reproduce the hierarchy among electron, muon and tau masses by letting the weights play the role of Froggatt-Nielsen charges. At level 5, our setup allows the treatment of left and right handed charged leptons on the same footing. We have optimized the free parameters of our models in order to match the experimental data, obtaining a good degree of compatibility and predictions for the absolute neutrino masses and the $C P$ violating phases. At a more fundamental level, the whole lepton sector could be correctly described by the simultaneous presence of several moduli. Our examples are meant to make a first step in this direction.

Keywords: Discrete Symmetries, Neutrino Physics

ARXIV EPRINT: 1908.11867 


\section{Contents}

1 Introduction 1

2 The models 3

2.1 Level 4 models 5

$\begin{array}{lll}2.2 & \text { Level } 5 \text { models } & 7\end{array}$

3 Results $\quad 9$

3.1 Fit to leptonic data 9

$\begin{array}{lll}3.2 & \text { Numerical results at level } 4 & 11\end{array}$

$\begin{array}{lll}3.3 & \text { Numerical results at level } 5 & 12\end{array}$

4 Conclusion $\quad 14$

$\begin{array}{ll}\text { A Finite modular group } \Gamma_{4} \text { and level } 4 \text { modular forms } & 16\end{array}$

$\begin{array}{ll}\text { B Finite modular group } \Gamma_{5} \text { and level } 5 \text { modular forms } & 17\end{array}$

$\begin{array}{lr}\text { C Numerical results in fundamental region } & 19\end{array}$

\section{Introduction}

Masses and mixing angles of elementary fermions are known with good precision and in the last few years the progress in the lepton sector has been particularly impressive, with neutrino squared mass differences and mixing angles that are attaining or approaching percent-level precision. Despite such an advance on the experimental side, the fundamental principle, if any, ruling this important aspect of fundamental interactions has remained elusive. In recent times a lot of attention has been focused on neutrinos, since the relatively mild mass hierarchy and the large mixing angles discovered through neutrino oscillations have not matched the expectations based on the knowledge of the quark sector. Neutrinos led to a change of perspective, particularly relevant when we look at the flavour puzzle in the light of a unified theory, where leptons and quarks loose their individuality.

One of the few tools we have to address the flavour puzzle is the one based on flavour symmetries, which, however, comes with its own drawbacks. Flavour symmetries cannot be exact symmetries [1] and Yukawa couplings are usually expressed as a power series in the symmetry breaking terms, with many independent free variables, to the detriment of predictability. In addition, such an approach typically makes use of several symmetry breaking parameters, with specific orientation in flavour space, considerably complicating the construction. Finally, the most popular flavour symmetries of the lepton sector constrain only mixing angles and phases, leaving fermion masses essentially undetermined [2-9]. 
Recently, modular invariance has been invoked as candidate flavour symmetry [10]. In its simplest implementation a unique complex field, the modulus, acts as symmetry breaking parameter, thus simplifying the vacuum alignment problem. Modular invariance, in the limit of exact supersymmetry, completely determines the Yukawa couplings, to any order of the expansion in powers of the modulus. Moreover, neutrino masses, mixing angles and phases are all related to each other and, in minimal models, depend only on a few parameters. The formalism has been extended to consistently include $C P$ transformations $[11]^{1}$ and it can involve several moduli $[16,17]$. The idea that Yukawa couplings are determined by a set of moduli is clearly not new, and has been naturally realized in the context of string theory [18-22], in D-brane compactification [23-29], in magnetized extra dimensions [30-32], and in orbifold compactification [33-36]. Modular invariance has also been incorporated in early flavour models [37-41]. However, the main advantage of the recent approach is that it can be implemented in a bottom-up perspective, relying on the group transformation properties of modular forms of given weight and level.

Several models of lepton masses and mixing angles have been built at level 2 [42, 43], $3[10,44-47], 4$ [48-50] and 5 [51, 52]. Extensions to quarks [53, 54] and to grand unified theories $[55,56]$ have also been proposed. In most of the existing constructions, there is a unique symmetry breaking parameter: the modulus itself. While this scenario is certainly appealing since it minimizes the symmetry breaking sector, it does not yet provide a convincing explanation of the charged lepton masses. The mass hierarchy is achieved by hand by introducing one parameter for each charged lepton species. This can be intuitively understood by recognizing that the dependence of modular forms on the modulus is nearly exponential and small neutrino mass hierarchies and large mixing angles require a modulus with small imaginary part, which is inadequate to generate the large hierarchies observed among electron, muon and tau masses. This may indicate that the charged lepton sector requires a different description, perhaps in terms of more moduli, a natural possibility in string theory.

In the present work we explore alternative descriptions of the charged lepton sector in a modular invariant framework. We test the dependence of charged lepton masses on an additional set of fields by including in the symmetry breaking sector both the modulus and ordinary flavons, chiral multiplets invariant under gauge transformations carrying nontrivial representations of the finite modular groups and non-trivial weights, to guarantee consistence with invariance under the full modular group. This has been done at level 3 in ref. [44] and at level 5 in ref. [52]. We will extend the investigation to level 4 and extend the possibilities studied so far al level 5. At level 4 the charged lepton Yukawa couplings are tailored to depend only on the flavons, with the hope of reproducing charged lepton masses with parameters similar in size, at least at the level of order of magnitudes. We will let right-handed charged leptons be responsible for the observed mass hierarchy, by assigning them different modular weights compensated by growing powers of the flavons, much as in refs. [39-41]. At level 5 we will take a more radical departure from the existing

\footnotetext{
${ }^{1}$ The interplay between $C P$ and modular invariance in string theory have been discussed in refs. [12, 13] and especially in refs. $[14,15]$ where a unified picture of flavour, $C P$ and modular invariance has been analyzed from a string theory perspective.
} 
constructions and we will assign the right-handed charged leptons to an irreducible triplet of $\Gamma_{5}$, to treat them more closely to their left-handed partners. In our models only the neutrino sector depends non-trivially on the modulus. As done in ref. [44], we will not attempt to dynamically select the vacuum configurations in the symmetry breaking sector. We have no compelling indications so far that Nature follows a dynamical principle to set the cosmological constant or the electroweak scale. We thus treat the vacuum expectation values (VEVs) as free parameters, to be varied to match the experimental data.

The models are built aiming at minimizing the number of free parameters. So far few predictive models use four independent parameters to describe neutrino masses, mixing angles and phases and a variety of models achieve that with five free parameters, including real and imaginary part of the modulus. As we will see the models we have been able to construct make use of at least five parameters and can be considered next-to-minimal. In our attempts we have also incorporated $C P$ invariance, to be spontaneously broken by the modulus and by the flavons. We present realistic examples where neutrino masse are described both in terms of the Weinberg operator and via the type I seesaw mechanism.

Our paper is organized as follows. In section 2 we briefly review the formalism of modular invariant supersymmetric theories applied to the lepton sector and we will describe our models. In section 3 we present the data, describe our fit and we show the results of the fit and the predictions of the models. Finally in 4 we draw our conclusion.

\section{The models}

We brefly review the formalism of modular invariant supersymmetric theories $[16,57]$. The models analyzed here are supersymmetric and gauge invariant under $\mathrm{SU}(3) \times \mathrm{SU}(2) \times \mathrm{U}(1)$. We are mainly interested to the Yukawa interactions, described by the action:

$$
\mathcal{S}=\int d^{4} x d^{2} \theta d^{2} \bar{\theta} K(\Phi, \bar{\Phi})+\int d^{4} x d^{2} \theta w(\Phi)+\text { h.c. }
$$

where $K(\Phi, \bar{\Phi})$, the Kähler potential, is a real gauge-invariant function of the chiral superfields $\Phi$ and their conjugates and $w(\Phi)$, the superpotential, is a holomorphic gauge-invariant function of the chiral superfields $\Phi$. The chiral superfields $\Phi=\left(\tau, \varphi^{(I)}\right)$ include the modulus $\tau$, a dimensionless chiral supermultiplet, and the remaining chiral supermultiplets, $\varphi^{(I)}$. Under the modular group $\Gamma$ the modulus transforms as

$$
\tau \rightarrow \gamma \tau \equiv \frac{a \tau+b}{c \tau+d}
$$

with $a, b, c$ and $d$ integers satisfying $a d-b c=1$. The modular group $\Gamma$ is an infinite discrete group, generated by the elements $S$ and $T$ satisfying $S^{2}=(S T)^{3}=1$. They act as

$$
\tau \rightarrow-\frac{1}{\tau} \quad(S) \quad \tau \rightarrow \tau+1 \quad(T) \quad .
$$

The transformation properties of $\varphi^{(I)}$ are fully specified by the data $\left(k_{I}, N, \rho^{(I)}\right)$, where $k_{I}$ (the weight) is a real number, $N$ (the level) is an integer and $\rho^{(I)}$ is a unitary representation 
of the quotient group $\Gamma_{N}=\Gamma / \Gamma(N) . \Gamma(N)$ is a principal congruence subgroup of $\Gamma$ and the level $N$ can be kept fixed in the construction. The multiplets $\varphi^{(I)}$ transform as

$$
\varphi^{(I)} \rightarrow(c \tau+d)^{k_{I}} \rho^{(I)}(\gamma) \varphi^{(I)}
$$

We choose a minimal form of the Kahler potential, invariant under (2.2), (2.4) up to Kahler transformations:

$$
K(\Phi, \bar{\Phi})=-h \log (-i \tau+i \bar{\tau})+\sum_{I}(-i \tau+i \bar{\tau})^{-k_{I}}\left|\varphi^{(I)}\right|^{2}
$$

where $h$ is a positive constant. Concerning the superpotential $w(\Phi)$, its expansion in power series of the supermultiplets $\varphi^{(I)}$ reads:

$$
w(\Phi)=\sum_{n} Y_{I_{1} \ldots I_{n}}(\tau) \varphi^{\left(I_{1}\right)} \ldots \varphi^{\left(I_{n}\right)}
$$

For the $n$-th order term to be modular invariant the functions $Y_{I_{1} \ldots I_{n}}(\tau)$ should be modular forms of weight $k_{Y}(n)$ and level $N$, transforming in the representation $\rho$ of $\Gamma_{N}$ :

$$
Y_{I_{1} \ldots I_{n}}(\gamma \tau)=(c \tau+d)^{k_{Y}(n)} \rho(\gamma) Y_{I_{1} \ldots I_{n}}(\tau)
$$

satisfying the conditions:

1. The weight $k_{Y}(n)$ should compensate the overall weight of the product $\varphi^{\left(I_{1}\right)} \ldots \varphi^{\left(I_{n}\right)}$ :

$$
k_{Y}(n)+k_{I_{1}}+\ldots+k_{I_{n}}=0
$$

2. The product $\rho \times \rho^{I_{1}} \times \ldots \times \rho^{I_{n}}$ contains an invariant singlet.

The above requirement is very restrictive. Indeed, for each level $N$ and for each even nonnegative weight $k$, there is only a finite number of linearly independent modular forms. ${ }^{2}$ They span the linear space $\mathcal{M}_{k}(\Gamma(N))$. Forms with vanishing weight are constant, that is independent from $\tau$. We will analyze models with $N=4$ and 5. The dimension of $\mathcal{M}_{k}(\Gamma(4))$ is $2 k+1$, while $\mathcal{M}_{k}(\Gamma(5))$ has dimension $5 k+1$. Modular forms of weight 2 generate the whole ring of modular forms. The five independent modular forms of level 4 and weight 2 have been constructed in ref. [48]. They decompose as $\mathbf{2}+\mathbf{3}^{\prime}$ under the finite group $\Gamma_{4} \equiv S_{4}$. The eleven independent modular forms of level 5 and weight 2 have been constructed in ref. [51] and [52]. They decompose as $\mathbf{3}+\mathbf{3}^{\prime}+\mathbf{5}$ under $\Gamma_{5} \equiv A_{5}$. In appendix A and B we list them.

The chiral multiplets $\varphi^{(I)}$ comprise three generations of lepton singlets $E^{c}$ and doublets $L$, the Higgses $H_{u, d}$, and gauge invariant flavons $\varphi$. We will consider both the case where neutrino masses arise through the Weinberg operator and the case where neutrinos get their masses through the seesaw mechanism. In the latter framework also three generations of gauge singlets $N^{c}$ are included. In our conventions both the modulus $\tau$ and the flavon $\varphi$ are dimensionless fields. The correct dimensions can be recovered by an appropriate rescaling.

\footnotetext{
${ }^{2}$ Recently modular forms of general integer weights and their transformation properties under the double covering of finite modular groups have been analyzed in ref. [58].
} 


\begin{tabular}{|c|c|c|c|c|c|c|c|c|}
\hline & $E_{1}^{c}$ & $E_{2}^{c}$ & $E_{3}^{c}$ & $N^{c}$ & $L$ & $H_{u, d}$ & $\varphi$ & $\varphi^{\prime}$ \\
\hline$\Gamma_{4} \equiv S_{4}$ & $\mathbf{1}$ & $\mathbf{1}$ & $\mathbf{1}$ & $\mathbf{3}$ & $\mathbf{3}$ & $\mathbf{1}$ & $\mathbf{3}$ & $\mathbf{1}^{\prime}$ \\
\hline$k_{I}$ (Seesaw) & $k-3 k_{\varphi}$ & $k-2 k_{\varphi}$ & $k-k_{\varphi}$ & $k$ & $-k$ & 0 & $k_{\varphi}$ & $k_{\varphi^{\prime}}$ \\
\hline$k_{I}$ (Weinberg) & $-k-3 k_{\varphi}$ & $-k-2 k_{\varphi}$ & $-k-k_{\varphi}$ & - & $k$ & 0 & $k_{\varphi}$ & $k_{\varphi^{\prime}}$ \\
\hline
\end{tabular}

Table 1. Chiral supermultiplets, transformation properties and weights. Weights for $E_{i}^{c}$ and $L$ depend on whether neutrinos get their masses from the seesaw mechanism or from the Weinberg operator. A possible choice leading to the superpotential given in the text is $k=-5 / 3, k_{\varphi^{\prime}}=+4 / 3$ and $k_{\varphi}=+3 / 2$. As a consequence, the neutrino sector depends only on $\varphi^{\prime}$ and the charged lepton sector depends only on $\varphi$.

Invariance under $C P$ can be incorporated in a consistent way [11] by requiring:

$$
\tau \stackrel{C P}{\longrightarrow}-\tau^{*},
$$

up to a modular transformation. On the chiral multiplets $\varphi^{(I)}$ a $C P$ transformation acts as

$$
\varphi^{(I)} \stackrel{C P}{\longrightarrow} X_{(I)}\left[\varphi^{(I)}\right]^{*},
$$

where $X_{(I)}$ is a matrix satisfying the consistency conditions:

$$
X_{(I)}\left[\rho^{(I)}(\gamma)\right]^{*} X_{(I)}^{-1}=\rho^{(I)}\left(\gamma^{\prime}\right) \quad, \quad\left(\gamma, \gamma^{\prime}\right) \in \Gamma \quad .
$$

In a basis where all the matrices $\rho^{(I)}(\gamma)$ are symmetric, these conditions are always solved by $X_{(I)}=\mathbb{1}$. This is the case of our choice of basis at level 5 . At level 4 our basis does not enjoy this property and a non-canonical solution for $X_{(I)}$ is listed in appendix A.

\section{$2.1 \quad$ Level 4 models}

The group $\Gamma_{4}$ has order 24 and is isomorphic to $S_{4}$. Its irreducible representations are $\mathbf{1}, \mathbf{1}^{\prime}, \mathbf{2}, \mathbf{3}$ and $\mathbf{3}^{\prime}$. It is generated by two elements $S$ and $T$ satisfying the relations $S^{2}=(S T)^{3}=T^{4}=\mathbb{1}$. In appendix A we detail the explicit form of the generators for the irreducible representations and the relevant Clebsch-Gordan coefficients used in this paper. The particle content, weights and representations of our models are shown in table 1 .

With the above assignment the superpotential reads

$$
w=w_{h}+w_{e}+w_{\nu},
$$

where $w_{h}, w_{e}, w_{\nu}$ describe the Higgs sector, the charged lepton sector and the neutrino sector, respectively. Since the Higgs sector plays no role in our discussion, we neglect $w_{h}$. We set $H_{u}=H_{d}=1$ in the superpotential, but we keep track of the correct dimension of the operators.

In the neutrino sector $w_{\nu}$ depends on the mass generation mechanism. When neutrino masses originate from the Weinberg operator we have:

$$
w_{\nu}=-\frac{1}{\Lambda}\left[\left(\varphi^{\prime} L L Y_{\mathbf{2}}\right)_{1}+\xi\left(\varphi^{\prime} L L Y_{\mathbf{3}^{\prime}}\right)_{1}\right],
$$




\begin{tabular}{cc}
\hline Weinberg, $\quad \mathcal{W}_{W}=\left(\begin{array}{ccc}0 & Y_{1} & -Y_{2} \\
Y_{1} & -Y_{2} & 0 \\
-Y_{2} & 0 & Y_{1}\end{array}\right)+\xi\left(\begin{array}{ccc}2 Y_{3} & -Y_{5} & -Y_{4} \\
-Y_{5} & 2 Y_{4} & -Y_{3} \\
-Y_{4} & -Y_{3} & 2 Y_{5}\end{array}\right)$ \\
\hline Seesaw, \\
$\mathcal{W}_{S}=\frac{y_{0}^{2}}{2}\left(\begin{array}{lll}1 & 0 & 0 \\
0 & 0 & 1 \\
0 & 1 & 0\end{array}\right) \mathcal{W}_{W}^{-1}\left(\begin{array}{lll}1 & 0 & 0 \\
0 & 0 & 1 \\
0 & 1 & 0\end{array}\right)$
\end{tabular}

Table 2. Relevant matrices in the neutrino sector of the superpotential in $\Gamma_{4}$ models.

where $\Lambda$ stands for the scale associated to lepton number violation, $(\ldots)_{r}$ denotes the $r$ representation of $\Gamma_{4}$ and $\xi$ is a free parameter. When light neutrinos get their masses from the seesaw mechanism, the terms of $w_{\nu}$ bilinear in the matter multiplets $L$ and $N^{c}$ read

$$
w_{\nu}=-y_{0}\left(N^{c} L\right)_{1}+\Lambda\left[\left(\varphi^{\prime} N^{c} N^{c} Y_{\mathbf{2}}\right)_{1}+\xi\left(\varphi^{\prime} N^{c} N^{c} Y_{\mathbf{3}^{\prime}}\right)_{1}\right]+\ldots
$$

Dots denote terms containing three or more powers of the matter fields, having no impact on our analysis. A truly minimal model would involve a single invariant in the neutrino sector. For instance, a suitable assignement of weights can allow the unique term $w_{\nu}=-\left(L L Y_{2}\right)_{1} / \Lambda$ (Weinberg) or $w_{\nu}=-\left(N^{c} N^{c} Y_{\mathbf{2}}\right)_{1} \Lambda$ (seesaw). We have studied these possibilities, but we found no viable choice of parameters which may reproduce data.

At energies below the mass scale $\Lambda$ for both models we have, in a matrix notation:

$$
w_{\nu}=-\frac{1}{\Lambda} L^{T} \mathcal{W} L+\ldots,
$$

where $\mathcal{W}$ denotes a matrix in generation space depending on the 5 independent level 4 and weight +2 modular forms $Y_{i}(\tau)(i=1, \ldots, 5)$. We list these results in table 2 , where the VEV of $\varphi^{\prime}$ has been absorbed in $\Lambda, Y_{i}$ stands for $Y_{i}(\tau)$, and the indices $W, S$ distinguish neutrino masses originating from the Weinberg operator or from the seesaw mechanism. The light neutrino mass matrix $m_{\nu}$ is

$$
m_{\nu}=\mathcal{W} \frac{v^{2}}{\Lambda} \sin ^{2} \hat{\beta}
$$

where $\tan \hat{\beta}$ is the ratio of VEVs, $\left\langle H_{u}\right\rangle /\left\langle H_{d}\right\rangle$. So far, the results in the neutrino sector would not vary had we instead defined $N^{c}$ and $L$ to transform as a $\mathbf{3}$ ', rather than a $\mathbf{3}$ under $\Gamma_{4}$. However, the following discussion in the charged lepton sector requires the properties as defined in table 1 . The superpotential $w_{e}$ for the charged lepton sector reads:

$$
w_{e}=-a E_{1}^{c}\left(L \varphi^{3}\right)_{1}-a^{\prime} E_{1}^{c}\left(L \varphi^{3}\right)_{1}^{\prime}-b E_{2}^{c}\left(L \varphi^{2}\right)_{1}-c E_{3}^{c}(L \varphi)_{1} \equiv-E^{c T} \mathcal{Y}_{e} L
$$

In the last equality we use a vector notation and

$$
\mathcal{Y}_{e}=\left(\begin{array}{ccc}
a\left(\varphi_{2}^{3}-2 \varphi_{1}^{3}+\varphi_{3}^{3}\right) & 3 a\left(\varphi_{1} \varphi_{2}^{2}-\varphi_{2} \varphi_{3}^{2}\right) & -3 a\left(\varphi_{2}^{2} \varphi_{3}-\varphi_{1} \varphi_{3}^{2}\right) \\
+a^{\prime}\left(\varphi_{1}^{3}+2 \varphi_{1} \varphi_{2} \varphi_{3}\right) & +a^{\prime}\left(\varphi_{1}^{2} \varphi_{3}+2 \varphi_{2} \varphi_{3}^{2}\right) & +a^{\prime}\left(\varphi_{1}^{2} \varphi_{2}+2 \varphi_{2}^{2} \varphi_{3}\right) \\
b\left(\varphi_{1}^{2}-\varphi_{2} \varphi_{3}\right) & b\left(\varphi_{2}^{2}-\varphi_{1} \varphi_{3}\right) & b\left(-\varphi_{1} \varphi_{2}+\varphi_{3}^{2}\right) \\
c \varphi_{1} & c \varphi_{3} & c \varphi_{2}
\end{array}\right)
$$


There are two independent $\Gamma_{4}$ invariants that can be built out of $L$ and $\varphi^{3}$, hence the two independent parameters $a$ and $a^{\prime}$. The dependence on the flavon supermultiplet $\varphi$ is fixed by the weight assignment. There is no dependence on the modulus $\tau$, since the bilinears $\left(E_{1}^{c} L, E_{2}^{c} L, E_{3}^{c} L\right)$ have weight $\left(-3 k_{\varphi},-2 k_{\varphi},-k_{\varphi}\right)$. Taking, for instance, $k_{\varphi}=+3 / 2$, these weights cannot be matched by modular forms. The charged lepton mass matrix $m_{e}$ reads

$$
m_{e}=\mathcal{Y}_{e} \frac{v}{\sqrt{2}} \cos \hat{\beta}
$$

Notice that if the flavon $\varphi$ is aligned along the $\left(0, \varphi_{2}, 0\right)$ direction, $\mathcal{Y}_{e}$ is diagonal and the charged lepton masses are given by:

$$
m_{e}=\frac{a}{\sqrt{2}} v \varphi_{2}^{3} \cos \hat{\beta} \quad, \quad m_{\mu}=\frac{b}{\sqrt{2}} v \varphi_{2}^{2} \cos \hat{\beta}, \quad m_{\tau}=\frac{c}{\sqrt{2}} v \varphi_{2} \cos \hat{\beta}
$$

Hence, a mass hierarchy can be generated by $\left|\varphi_{2}\right|<1$, even with $a, b$ and $c$ of the same order.

In our numerical analysis we will treat the modulus $\tau$ and the VEV of $\varphi$ as free parameters. Beyond that, the parameters controlling lepton masses and mixing angles are the overall scale $\Lambda$ and the five dimensionless constants $\xi, a, a^{\prime}, b$ and $c$. Without loss of generality, we can require $a, a^{\prime}, b$ and $c$ to be real, since their phases are always unphysical. On the contrary, the phase of $\xi$ cannot be removed by a field redefinition. We will consider two options, either requiring the theory to be invariant under $C P$ at the Lagrangian level, or not. In the former case, using the $C P$ transformation given in appendix $\mathrm{A}$, we find that $\xi$ should be real and $C P$ can be spontaneously broken by the VEVs of $\tau$ and/or $\varphi$. In the latter case, we will treat $\xi$ as a complex free parameter. The dependence on $\tan \hat{\beta}$ can be absorbed into the above parameters and will not be explicitly shown when reporting numerical values.

\subsection{Level 5 models}

The irreducible representations of the group $\Gamma_{5} \equiv A_{5}$ are $\mathbf{1}, \mathbf{3}, \mathbf{3}^{\prime}, \mathbf{4}$ and $\mathbf{5}$. Its generators are $S$ and $T$, satisfying $S^{2}=(S T)^{3}=T^{5}=\mathbb{1}$. In appendix B we specify the explicit form of the generators for each representation, together with the relevant Clebsh-Gordan coefficients. Here, we construct modular-invariant models in which all leptons are collected into 3 or $3^{\prime}$ multiplets of $A_{5}$, containing the three generations of each type of field. We take the neutrino sector to be minimal, it should only depend on the modulus $\tau$ and an overall scale. Modular forms will not appear in the charged-lepton sector, which instead will contain two extra flavons. In table 3, we show the assignments of representations and weights that we consider.

Setting $H_{u}=H_{d}=1$, the neutrino sector $w_{\nu}$ of the superpotential is, depending on the choice Weinberg vs. Seesaw and $\left(\rho_{L}=\rho_{N}\right)$ vs. $\left(\rho_{L} \neq \rho_{N}\right)$ :

$$
w_{\nu}= \begin{cases}-\frac{1}{\Lambda}\left(L L Y_{5}\right)_{1} & \text { Weinberg } \\ -y_{0}\left(N^{c} L\right)_{1}+\Lambda\left(N^{c} N^{c} Y_{\mathbf{5}}\right)_{1} & \text { Seesaw, } \rho_{L}=\rho_{N} \\ -y_{0}\left(N^{c} L Y_{\mathbf{5}}\right)_{1}+\Lambda\left(N^{c} N^{c}\right)_{1} & \text { Seesaw, } \rho_{L} \neq \rho_{N}\end{cases}
$$

The case of $\rho_{L} \sim \rho_{N} \sim 3$ has been studied in detail in ref. [62] and so not discussed here. 


\begin{tabular}{|c|c|c|c|c|c|c|}
\hline & $E^{c}$ & $N^{c}$ & $L$ & $H_{u, d}$ & $\varphi$ & $\chi$ \\
\hline$\Gamma_{5} \equiv A_{5}$ & $\rho_{L}$ & $\rho_{N}$ & $\rho_{L}$ & 1 & $\rho_{L}$ & 1 \\
\hline$k_{I}$ & $-3-k_{L}$ & $k_{N}$ & $k_{L}$ & 0 & $3 / 2$ & $3 / 2$ \\
\hline
\end{tabular}

\begin{tabular}{|c|cc|cc|}
\hline & $\rho_{N}$ & $\rho_{L}$ & $k_{N}$ & $k_{L}$ \\
\hline Weinberg & - & $\mathbf{3}$ & - & -1 \\
& - & $\mathbf{3}^{\prime}$ & - & -1 \\
\hline \multirow{5}{*}{ Seesaw } & $\mathbf{3}$ & $\mathbf{3}$ & -1 & 1 \\
& $\mathbf{3}^{\prime}$ & $\mathbf{3}^{\prime}$ & -1 & 1 \\
& $\mathbf{3}$ & $\mathbf{3}^{\prime}$ & 0 & -2 \\
& $\mathbf{3}^{\prime}$ & $\mathbf{3}$ & 0 & -2 \\
\hline
\end{tabular}

Table 3. Chiral supermultiplets, transformation properties and weights for the level-5 models.

\begin{tabular}{|c|c|c|c|c|c|c|}
\hline $\begin{array}{c}\text { Weinberg, } \rho_{L}=\mathbf{3} \\
k_{L}=-1\end{array}$ & & $\mathcal{W}=$ & $\begin{array}{c}2 Y_{1} \\
-\sqrt{3} Y_{5} \\
-\sqrt{3} Y_{2}\end{array}$ & $\begin{array}{l}\sqrt{3} Y_{5} \\
\sqrt{6} Y_{4} \\
-Y_{1} \\
\end{array}$ & $\left.\begin{array}{c}-\sqrt{3} Y_{2} \\
-Y_{1} \\
\sqrt{6} Y_{3}\end{array}\right)$ & \\
\hline $\begin{array}{c}\text { Weinberg, } \rho_{L}=\mathbf{3}^{\prime} \\
k_{L}=-1\end{array}$ & & $\mathcal{W}=$ & $\begin{array}{c}2 Y_{1} \\
-\sqrt{3} Y_{4} \\
-\sqrt{3} Y_{3}\end{array}$ & $\begin{array}{l}\sqrt{3} Y_{4} \\
\sqrt{6} Y_{2} \\
-Y_{1}\end{array}$ & $\left.\begin{array}{c}-\sqrt{3} Y_{3} \\
-Y_{1} \\
\sqrt{6} Y_{5}\end{array}\right)$ & \\
\hline $\begin{array}{c}\text { Seesaw, } \rho_{L}=\rho_{N}=\mathbf{3} \\
k_{L}=1, k_{N}=-1\end{array}$ & $\mathcal{W}_{W}=$ & $\begin{array}{c}2 Y_{1} \\
-\sqrt{3} Y_{5} \\
-\sqrt{3} Y_{2}\end{array}$ & $\begin{array}{cc} & -\sqrt{3} Y_{5} \\
5 & \sqrt{6} Y_{4} \\
2 & -Y_{1}\end{array}$ & $\begin{array}{c}-\sqrt{3} Y_{2} \\
-Y_{1} \\
\sqrt{6} Y_{3}\end{array}$ &, $\mathcal{Y}_{\nu}=$ & $\left(\begin{array}{lll}1 & 0 & 0 \\
0 & 0 & 1 \\
0 & 1 & 0\end{array}\right)$ \\
\hline $\begin{array}{c}\text { Seesaw, } \rho_{L}=\rho_{N}=\mathbf{3}^{\prime} \\
k_{L}=1, k_{N}=-1\end{array}$ & $\mathcal{W}_{W}=$ & $\begin{array}{c}2 Y_{1} \\
-\sqrt{3} Y_{4} \\
-\sqrt{3} Y_{3}\end{array}$ & $\begin{array}{c}-\sqrt{3} Y_{4}- \\
\sqrt{6} Y_{2} \\
3 \\
3\end{array}$ & $\begin{array}{c}-\sqrt{3} Y_{3} \\
-Y_{1} \\
\sqrt{6} Y_{5}\end{array}$ &, $\mathcal{Y}_{\nu}=$ & $\left(\begin{array}{lll}1 & 0 & 0 \\
0 & 0 & 1 \\
0 & 1 & 0\end{array}\right)$ \\
\hline $\begin{array}{c}\text { Seesaw, } \rho_{L}=3, \rho_{N}=\mathbf{3}^{\prime} \\
k_{L}=-2, k_{N}=0\end{array}$ & $\mathcal{W}_{W}=$ & $\left.\begin{array}{lll}1 & 0 & 0 \\
0 & 0 & 1 \\
0 & 1 & 0\end{array}\right)$ & $\int, \mathcal{Y}_{\nu}=$ & $\begin{array}{c}\sqrt{3} Y_{1} \\
Y_{4} \\
Y_{3} \\
\end{array}$ & $\begin{array}{c}Y_{5} \\
-\sqrt{2} Y_{3} \\
-\sqrt{2} Y_{2} \\
\end{array}$ & $\left.\begin{array}{c}Y_{2} \\
-\sqrt{2} Y_{5} \\
-\sqrt{2} Y_{4}\end{array}\right)$ \\
\hline $\begin{array}{c}\text { Seesaw, } \rho_{L}=3^{\prime}, \rho_{N}=\mathbf{3} \\
k_{L}=-2, k_{N}=0\end{array}$ & $\mathcal{W}_{W}=$ & $\left(\begin{array}{lll}1 & 0 & 0 \\
0 & 0 & 1 \\
0 & 1 & 0\end{array}\right)$ & $\mathcal{Y}_{\nu}=$ & $\begin{array}{c}\sqrt{3} Y_{1} \\
Y_{5} \\
Y_{2}\end{array}$ & $\begin{array}{c}Y_{4} \\
-\sqrt{2} Y_{3} \\
-\sqrt{2} Y_{5}\end{array}$ & $\left.\begin{array}{c}Y_{3} \\
-\sqrt{2} Y_{2} \\
-\sqrt{2} Y_{4}\end{array}\right)$ \\
\hline
\end{tabular}

Table 4. Relevant matrices in the neutrino sector of the superpotential in $\Gamma_{5}$ models.

Below the energy scale $\Lambda, w_{\nu}$ can always be written as

$$
w_{\nu}=-\frac{1}{\Lambda} L^{T} \mathcal{W} L
$$

with $\mathcal{W}$ a $3 \times 3$ matrix, whose explicit form for each case can be read from table 4 , using the equation $\mathcal{W}=\frac{y_{0}^{2}}{2} \mathcal{Y}_{\nu}^{T} \mathcal{W}_{W}^{-1} \mathcal{Y}_{\nu}$ for the seesaw case. The light neutrino mass matrix $m_{\nu}$ can be obtained from $\mathcal{W}$ as in eq. 2.16.

The charged-lepton sector $w_{e}$ of the superpotential is

$$
w_{e}=\alpha\left(E^{c} L\right)_{\mathbf{1}} \chi^{2}+\beta\left(E^{c} L\right)_{\mathbf{3}} \chi \varphi+\gamma\left(E^{c} L\right)_{\mathbf{5}}\left(\varphi^{2}\right)_{\mathbf{5}}+\delta\left(E^{c} L\right)_{\mathbf{1}}\left(\varphi^{2}\right)_{\mathbf{1}} \equiv-E^{c T} \mathcal{Y}_{e} L
$$

In what follows we set the flavons to their vevs and denote them by $\chi, \varphi_{i}$. We absorb $\chi \neq 0, \varphi_{1} \neq 0$ and the Lagrangian parameter $\delta$ into $\alpha, \beta, \gamma, \varphi_{2}$ and $\varphi_{3}$. Once this is done, 
the matrix $\mathcal{Y}_{e}$ takes the form

$$
\mathcal{Y}_{e}=\left(\begin{array}{ccc}
\alpha+4 \gamma\left(1-\varphi_{2} \varphi_{3}\right) & (\beta+6 \gamma) \varphi_{3} & (-\beta+6 \gamma) \varphi_{2} \\
(-\beta+6 \gamma) \varphi_{3} & 6 \gamma \varphi_{3}^{2} & \alpha+\beta-2 \gamma\left(1-\varphi_{2} \varphi_{3}\right) \\
(\beta+6 \gamma) \varphi_{2} & \alpha-\beta-2 \gamma\left(1-\varphi_{2} \varphi_{3}\right) & 6 \gamma \varphi_{2}^{2}
\end{array}\right)
$$

The charged lepton mass matrix $m_{e}$ has the same form as in eq. (2.19), $m_{e}=\mathcal{Y}_{e} v \cos \hat{\beta} / \sqrt{2}$. Setting $\varphi_{2}=\varphi_{3}=0$ and switching the last two rows gives a diagonal $m_{e}$, with eigenvalues $m_{a}=(\alpha+4 \gamma) \frac{v}{\sqrt{2}} \cos \hat{\beta} \quad, \quad m_{b}=(\alpha-\beta-2 \gamma) \frac{v}{\sqrt{2}} \cos \hat{\beta} \quad, \quad m_{c}=(\alpha+\beta-2 \gamma) \frac{v}{\sqrt{2}} \cos \hat{\beta}$.

As for the $\Gamma_{4}$ case, we treat $\tau$ and the $\operatorname{VEVs} \varphi_{2,3}$ as parameters to be freely varied in our fit. The remaining parameters are the overall scale $\Lambda$ and the dimensionless constants $\alpha, \beta$ and $\gamma$. By enforcing $C P$ conservation, the latter three are required to be real. The dependence on $\tan \hat{\beta}$ can be absorbed into these parameters.

\section{Results}

In this section we identify which scenarios we analyse, state the experimental data used and report the results of a chi-square analysis with the predictions of the models. In table 5, we list the seven scenarios which reproduce the data well, with a reasonable $\chi_{\min }^{2}$ and minimum number of parameters. We will present results only for these scenarios, omitting those presenting a high $\chi_{\min }^{2}$ or a large number of parameters. We identify the different cases with a code referring to the modular level $\Gamma_{4} \equiv S_{4}$ or $\Gamma_{5} \equiv A_{5}$ "4 (5)"; Weinberg or Seesaw "W (S)"; $C P$ conserving or violating "C (V)". For the $A_{5}$ Weinberg scenario, we add the transformation property of the lepton triplet, whether this transforms as a $\mathbf{3}$, or 3' "3 (3p)".

We present the results in this section for which $\tau$ is not restricted to be in the fundamental domain, $|\operatorname{Re}(\tau)| \leq 1 / 2,|\tau| \geq 1$. However, in appendix $\mathrm{C}$ we also include a full list of modular transformations to the set of input parameters which transforms $\tau$ into the fundamental region, as well as the explicit numerical values for these transformed parameters, which will yield the same set of physical observables. In this main text we list the non-fundamental region input parameters to avoid confusion stemming from spurious additional imaginary parameters which are just an artefact of a basis transformation.

\subsection{Fit to leptonic data}

In table 6 , we list the experimental data and errors we use to calculate our pulls and $\chi_{\min }^{2}$ values. For the Yukawa couplings, we use the renomalised values at $m_{Z}$ scale, as detailed in ref. [59]. For the neutrino oscillation data, we use the most recent results from the NuFit collboration, ref. [60]. For the calculation of our $\chi_{\min }^{2}$, we assume the conservative estimate of gaussian errors, unless explicitly stated otherwise. Even though current data seem to prefer normal to inverted neutrino mass ordering, we do not weight this option in our $\chi^{2}$ function. 


\begin{tabular}{|c|c|c|c|c|}
\hline Model & Operator & $C P$ conservation & Charged Lepton sector & Case Identifier \\
\hline$S_{4}$ & Weinberg & $C P$ & Diagonal & $4 \mathrm{WV}$ \\
$S_{4}$ & Seesaw & $C P$ & Diagonal & $4 \mathrm{SV}$ \\
$S_{4}$ & Weinberg & $C P$ & Modified & $4 \mathrm{WC}$ \\
$S_{4}$ & Seesaw & $C P$ & Modified & $4 \mathrm{SC}$ \\
\hline$A_{5}$ & Weinberg, $\rho_{L}=\mathbf{3}$ & $C P$ & Modified & $5 \mathrm{WC} 3$ \\
$A_{5}$ & Weinberg, $\rho_{L}=\mathbf{3}^{\prime}$ & $C P$ & Modified & $5 \mathrm{WC} 3 \mathrm{p}$ \\
$A_{5}$ & Seesaw, & $C P$ & Modified & $5 \mathrm{SC}$ \\
& $\rho_{L}=\mathbf{3}, \rho_{N}=\mathbf{3}^{\prime}, \operatorname{Im}\left(\varphi_{2,3}\right)=0$ & & & \\
\hline
\end{tabular}

Table 5. A list of the seven scenarios presented with good fits to data.

\begin{tabular}{|l|l|}
\hline$y_{e}\left(m_{Z}\right)$ & $2.794745(16) \times 10^{-6}$ \\
\hline$y_{\mu}\left(m_{Z}\right)$ & $5.899863(19) \times 10^{-4}$ \\
\hline$y_{\tau}\left(m_{Z}\right)$ & $1.002950(91) \times 10^{-2}$ \\
\hline
\end{tabular}

\begin{tabular}{|c|c|c|}
\hline & $\mathrm{IO}$ & $\mathrm{NO}$ \\
\hline$\frac{\Delta m_{21}^{2}}{10^{-5} \mathrm{eV}^{2}}$ & $7.39(21)$ & $7.39(21)$ \\
\hline$\frac{\Delta m_{3 \ell}^{2}}{10^{-3} \mathrm{eV}^{2}}$ & $-2.512(33)$ & $+2.525(32)$ \\
\hline $\sin ^{2} \theta_{12}$ & $0.310(13)$ & $0.310(13)$ \\
\hline $\sin ^{2} \theta_{13}$ & $0.02263(66)$ & $0.02240(66)$ \\
\hline $\sin ^{2} \theta_{23}$ & $0.582(17)$ & $0.582(17)$ \\
\hline$\delta / \pi$ & $1.56(15)$ & $1.21(19)$ \\
\hline
\end{tabular}

Table 6. Left panel: charged lepton Yukawa couplings renormalized at the $m_{Z}$ scale, from ref. [59]. Right panel: neutrino oscillation data, from ref. [60]. The squared mass difference $\Delta m_{3 \ell}^{2}$ is equal to $\Delta m_{31}^{2}$ for normal ordering and $\Delta m_{32}^{2}$ for inverted ordering. Errors, shown in brackets, are the average of positive and negative $1 \sigma$ deviations. The $\chi^{2}$ function is not gaussian along $\operatorname{the}^{2} \sin ^{2} \theta_{23}$ direction and our definition overestimates the error.

We show our results for all the considered $\Gamma_{4}$ and $\Gamma_{5}$ cases in the three tables contained in table 7 and 8 respectively. For each case we present the point in parameter space which minimises the $\chi^{2}$, as a result of a numerical minimisation procedure. In the first table, one finds the predictions and, in parentheses, pulls to the six observed neutrino parameters: the two mass squared differences, $\Delta m_{21}^{2}, \Delta m_{3 l}^{2}$ (where the latter refers to $\Delta m_{32}^{2}>0$ for NO and $\Delta m_{31}^{2}<0$ for IO), three PMNS angles, $\theta_{12}, \theta_{13}, \theta_{23}$, and $C P$ violating phase, $\delta$; as well as the final $\chi_{\min }^{2}$. In the second table, we list the predictions for each scenario for the: three individual neutrino masses, $m_{1}, m_{2}, m_{3}$; Majorana phases $\alpha_{21}, \alpha_{31}$; neutrinoless double beta decay parameter, $m_{e e}$; and Mass Ordering (MO). In the third table we specify the input parameters used to generate the best fit point discussed. In neither $\Gamma_{4}$, nor $\Gamma_{5}$ do we present the pulls from the Yukawa of the charged lepton sector, as we find sufficient freedom for every considered case to reproduce the observed values with negligible pulls $\left(\Delta \chi^{2}<0.01\right)$. 


\subsection{Numerical results at level 4}

To minimize the number of effective parameters, we first analyze the case of diagonal charged lepton sector. This can be realized by fixing the VEV of the flavon $\varphi$ along the direction $\left(0, \varphi_{2}, 0\right)$. All terms depending on $a^{\prime}$ drop. The remaining input parameters $a, b$ and $c$, can be fixed to exactly reproduce the charged lepton masses:

$$
(a, b, c)=\frac{\sqrt{2}}{v \cos \beta}\left(\frac{m_{e}}{\varphi_{2}^{3}}, \frac{m_{\mu}}{\varphi_{2}^{2}}, \frac{m_{\tau}}{\varphi_{2}}\right) .
$$

Due to the hierarchical pattern in powers of the VEV, these input parameters may be all of similar order by fixing, for example, $\left|\varphi_{2}\right|=1 / 100$, which leads to

$$
a \cos \beta \simeq 2.8, \quad b \cos \beta \simeq 5.9, \quad c \cos \beta \simeq 1 .
$$

We are left with 3 Lagrangian parameters, $(\Lambda, \operatorname{Re}(\xi), \operatorname{Im}(\xi))$ and the (complex) modulus VEV $\tau$. Choosing the neutrino mass generated by the Weinberg operator (denoted case "4WV"), we get a good agreement between the model and the data by the parameter choice shown in table 7 , with a $\chi_{\min }^{2} \sim 0.6$. We also present results for the same scenario, but now with neutrino mass generated by a type-I seesaw (denoted case "4SV"), with a $\chi_{\min }^{2} \sim 1.1$.

We may further reduce the number of free parameters by imposing that the Lagrangian be $C P$ conserving. This amounts, in our basis, to requiring real Lagrangian parameters, i.e. $\operatorname{Im}(\xi)=0$. We found no feasible solutions with this further restriction keeping the charged lepton sector diagonal as before. Thus we relax this requirement and at the same we set $a^{\prime}=0$. Though $a^{\prime}$ is a legitimate parameter of our model, we can safely neglect it in the limit of nearly diagonal charged lepton sector. Indeed, in such a limit, the contribution to lepton mixing is dominated by the elements of $\mathcal{Y}_{e}$ below the diagonal, controlled by the parameters $b$ and $c$. We find a good fit to data allowing small perturbations (in units of $\varphi_{2}$ ) of $\operatorname{Im}\left(\varphi_{1}\right)=-\operatorname{Im}\left(\varphi_{3}\right) \neq 0$. Along this particular direction $C P$ is spontaneously broken, see appendix A, and the charged lepton sector contributes to the physical phases of the PMNS matrix. However, the main motivation for choosing such a direction is to show that it is possible to achieve a good agreement with the data by turning on a minimum number of extra parameters. We present our results for this scenario for both the Weinberg case (denoted " $4 \mathrm{WC}$ "), with $\chi_{\min }^{2} \sim 3.2$ and the seesaw case (denoted "4SC"), with $\chi_{\min }^{2} \sim 0.3$. In both $C P$ conserving and violating scenarios, neutrino masses from the Weinberg operator have inverted ordering, while those coming from the seesaw mechanism are normal ordered.

In our setup we were unable to describe both the neutrino masses and the mixing matrix with fewer than five parameters. On the other hand the overall results and predictions are quite stable with respect to the details of the model. The quality of the fit is quite similar in all cases analysed and the results mainly depend on the choice between the Weinberg operator and the seesaw mechanism. In both cases the neutrino mass spectrum is nearly degenerate and the lightest neutrino mass is around $40 \mathrm{meV}$. When we adopt the Weinberg operator (seesaw mechanism) $m_{e e}$ is close to 60 (40) meV. A normally ordered spectrum (corresponding to the seesaw mechanism) predicting a relatively high $m_{e e}$ parameter seems a common feature to most of the models enjoying modular invariance and providing a good 


\begin{tabular}{|c|c|c|c|c|c|c|c|}
\hline & \multicolumn{6}{|c|}{ value (pull) } & \\
\hline Case & $\Delta m_{21}^{2} \cdot 10^{5} \mathrm{eV}^{-2}$ & $\Delta m_{3 l}^{2} \cdot 10^{3} \mathrm{eV}^{-2}$ & $\sin ^{2} \theta_{12}$ & $\sin ^{2} \theta_{13}$ & $\sin ^{2} \theta_{23}$ & $\delta / \pi$ & $\chi_{\min }^{2}$ \\
\hline $4 W V$ & $7.39(0)$ & $-2.517(-0.2)$ & $0.310(+0.0)$ & $0.02262(-0.0)$ & $0.583(+0.1)$ & $1.68(+0.8)$ & 0.6 \\
\hline $4 S V$ & $7.39(0)$ & $2.527(+0.1)$ & $0.310(+0.0)$ & $0.02241(+0.0)$ & $0.580(-0.1)$ & $1.40(+1.0)$ & 1.1 \\
\hline $4 W C$ & $7.39(0)$ & $-2.512(-0.0)$ & $0.310(+0.0)$ & $0.02264(+0.0)$ & $0.580(-0.1)$ & $1.83(+1.8)$ & 3.2 \\
\hline $4 S C$ & $7.39(0)$ & $2.526(+0.0)$ & $0.317(+0.5)$ & $0.02237(-0.1)$ & $0.580(-0.1)$ & $1.25(+0.2)$ & 0.3 \\
\hline
\end{tabular}

\begin{tabular}{|c|c|c|c|c|c|c|c|}
\hline & \multicolumn{7}{|c|}{ value } \\
\hline Case & $m_{1} \cdot 10^{2} \mathrm{eV}^{-2}$ & $m_{2} \cdot 10^{2} \mathrm{eV}^{-2}$ & $m_{3} \cdot 10^{2} \mathrm{eV}^{-2}$ & $\alpha_{21} / \pi$ & $\alpha_{31} / \pi$ & $m_{e e} \cdot 10^{2} \mathrm{eV}^{-1}$ & MO \\
\hline $4 W V$ & 6.56 & 6.61 & 4.31 & 0.21 & 1.76 & 6.18 & IO \\
\hline $4 S V$ & 4.23 & 4.32 & 6.57 & 0.22 & 0.54 & 4.01 & NO \\
\hline $4 W C$ & 6.33 & 6.39 & 3.96 & 1.88 & 1.69 & 6.20 & IO \\
\hline $4 S C$ & 4.26 & 4.35 & 6.59 & 0.11 & 0.30 & 4.25 & NO \\
\hline
\end{tabular}

\begin{tabular}{|c|c|c|c|c|c|c|c|c|c|}
\hline & \multicolumn{10}{|c|}{ Input parameters } \\
\hline Case & $\operatorname{Re}(\tau)$ & $\operatorname{Im}(\tau)$ & $\operatorname{Re}(\xi)$ & $\operatorname{Im}(\xi)$ & $\operatorname{Im}\left(\varphi_{3}\right)=-\operatorname{Im}\left(\varphi_{1}\right)$ & $a$ & $b$ & $c$ & $1 / \Lambda\left(\mathrm{eV}^{-1}\right)$ \\
\hline $4 W V$ & 1.155 & 0.9797 & -2.536 & -0.07654 & - & 2.795 & 5.900 & 1.003 & 0.007395 \\
\hline $4 S V$ & 0.8436 & 0.9968 & -2.600 & 0.1151 & - & 2.795 & 5.900 & 1.003 & 0.7672 \\
\hline $4 W C$ & 2.530 & 0.5380 & -0.1063 & - & -0.001063 & 2.647 & 5.899 & 0.9918 & 0.003799 \\
\hline $4 S C$ & 2.506 & 0.5905 & -2.595 & - & 0.001081 & 2.642 & 5.899 & 0.9914 & 1.301 \\
\hline
\end{tabular}

Table 7. Results of the fit to lepton data for the $\Gamma_{4}$ models. In the top panel, best values and pulls for the observables used in the fit. Also the minimum $\chi^{2}$ is shown. In the middle table, predictions of the models: neutrino masses, phases and parameter $m_{e e}$ relevant for neutrinoless double beta decay. In the bottom panel input parameters at the minimum of the $\chi^{2}$ function. We have fixed $\varphi_{2}=0.01$ for all four cases. To simplify the notation, the factors $\cos \hat{\beta}$ and $1 / \sin ^{2} \hat{\beta}$ have been omitted from $a, b, c$ and $\Lambda$, respectively.

fit to the data. The neutrino masses in our model are slightly heavier than those of the level 4 models studied in refs. [48, 49].

\subsection{Numerical results at level 5}

We now turn to the models at level 5. Unlike in level 4, all the examples listed here produce a $C P$ conserving Lagrangian. In our basis, this requirement is that all Lagrangian parameters be real. The charged lepton masses are essentially controlled by $\alpha, \beta$, $\gamma$, while neutrino masses and mixing angles are mainly governed by $\Lambda, \tau$ and $\varphi$. We fix $\varphi_{1}=1$ and, to reduce the number of parameters, we restrict the two VEVs of $\left(\varphi_{2}, \varphi_{3}\right)$ to real values. Neutrino properties are thus described by a total of five parameters and $C P$ violating phases are entirely due to the neutrino sector, since $C P$ is preserved by a real $\varphi$ VEV. Here again this choice is mainly dictated by the desire to match the data using a small number of parameters.

As we can see from table 8 , we get the best agreement with data when neutrino masses come from the Weinberg operator, with $\rho_{L} \sim \mathbf{3}$ (denoted case "5WC3"), for which we get a $\chi_{\min }^{2} \sim 1.1$. The $\tau$ value is very close to the border of the fundamental region (see also table 10 in appendix $\mathrm{C}$ ), where $C P$ is conserved. This result strongly supports the 
indication that, in a $C P$ invariant model, even a tiny departure from the region of moduli space where $C P$ is preserved can cause large observable $C P$-violating effects [11]. We also notice that all the components of the multiplet $\varphi$ are of the same order, indicating that the charged lepton mass matrix is far from the diagonal form, related to $\varphi \propto(1,0,0)$. This is a new feature, since in the level 4 models discussed here and in the level 3 model of ref. [44], the contribution to the lepton mixing of the charged lepton mass matrix (depending on ordinary flavons) is small. The model predicts $m_{e e} \approx 27 \mathrm{meV}$. The mass ordering is inverted, as in all previous cases dealing with the Weinberg operator. An exception is provided by the other Weinberg case at level 5 in which $\rho_{L} \sim \mathbf{3}^{\prime}$ (denoted "5WC3p"), which predicts normal ordering at the price of a considerably worse $\chi_{\min }^{2} \sim 12.6$. The largest pulls are the one in $\delta$, which deviates by more than $3 \sigma$ and by $\sin ^{2} \theta_{13}$, about $1 \sigma$ below the current best value.

We have also explored this model in a seesaw scenario, in which $\rho_{L} \sim \mathbf{3}, \rho_{N} \sim \mathbf{3}^{\prime}$ (denoted "5SC"). The agreement with data is not excellent and our estimate of the $\chi_{\min }^{2}$ is 11.1. The main contributions to the $\chi_{\min }^{2}$ come from $\delta$, which deviates by more than $2 \sigma$ and by $\sin ^{2} \theta_{23}$, about $2 \sigma$ below the current best value. For $\sin ^{2} \theta_{23} \simeq 0.45$ we do not use the nominal pull, since the error is non-gaussian. We assess the contribution to the $\chi_{\min }^{2}$ directly using the results from NuFit. The neutrino mass spectrum has normal ordering. Specific to the seesaw realization are the prediction of $\theta_{23}$ in the first octant and of a vanishing $m_{1}$. The latter result has no counterpart in any model based on modular invariance so far investigated. The presence of a vanishing eigenvalue is independent from the choice of the modular parameter $\tau$ and is due to the fact that the determinant of the combination $\mathcal{Y}_{\nu}^{T} \mathcal{W}_{W}^{-1} \mathcal{Y}_{\nu}$ vanishes identically for the chosen representations. Though we do not have a full analytic proof of such behavior, we have checked it by means a $q$-expansion of the modular forms $Y_{i}(i=1, \ldots, 5)$ : the determinant is proportional to a power of $q$ that grows with the order at which we stop the $q$-expansion of $Y_{i}$. The vanishing of the determinant probably reflects one of the many algebraic identities involving lowest weight modular forms. As a consequence $m_{e e} \approx 1.3 \mathrm{meV}$ is rather small.

In all these cases we find that the spread of the parameters $\alpha, \beta, \gamma$ is less than one order of magnitude, much less than the one among the charged lepton masses. This statement requires a specification since, from the matrix of charged lepton Yukawa couplings, eq. (2.24), for $\alpha, \beta, \gamma$ of the same order and generic VEVs $\varphi_{i}(i=1,2,3)$, there is no evident preferred pattern of eigenvalues. Indeed, though the best fit values of $\alpha, \beta, \gamma$ are of the same order, some amount of tuning is needed to correctly reproduce the masses. This can be appreciated from eq. (2.25), the unrealistic case of diagonal $\mathcal{Y}_{e}$. Indeed, close to our best fit point, the combinations $\alpha+4 \gamma, \alpha-\beta-2 \gamma$ and $\alpha+\beta-2 \gamma$ are of order $1,0.01$ and 10 respectively, revealing a hidden conspiracy of the input parameters. Our approach and the related results significantly differ from those of refs. [51, 52] where several modular invariant models at level 5 have been analysed, under the assumption that the charged lepton sector be always diagonal [51] or diagonal when depending on ordinary flavons [52]. We have also looked for a better agreement with data in the seesaw case by relaxing the requirement of a real $\left(\varphi_{2}, \varphi_{3}\right)$. At the price of more parameters, we obtain an better fit to data, though we do not present this example explicitly. 


\begin{tabular}{|c|c|c|c|c|c|c|c|}
\hline & \multicolumn{6}{|c|}{ value (pull) } & \\
\hline Case & $\Delta m_{21}^{2} \cdot 10^{5} \mathrm{eV}^{-2}$ & $\Delta m_{3 l}^{2} \cdot 10^{3} \mathrm{eV}^{-2}$ & $\sin ^{2} \theta_{12}$ & $\sin ^{2} \theta_{13}$ & $\sin ^{2} \theta_{23}$ & $\delta / \pi$ & $\chi_{\min }^{2}$ \\
\hline $5 W C 3$ & $7.39(0)$ & $-2.512(+0.0)$ & $0.312(+0.1)$ & $0.02260(-0.0)$ & $0.592(+0.6)$ & $1.69(+0.9)$ & 1.1 \\
\hline $5 W C 3 p$ & $7.39(0)$ & $2.525(+0.0)$ & $0.309(-0.1)$ & $0.0217(-1.2)$ & $0.586(+0.3)$ & $0.57(-3.3)$ & 12.6 \\
\hline $5 S C$ & $7.39(0)$ & $2.522(-0.1)$ & $0.292(-1.4)$ & $0.0228(+0.5)$ & $0.449\left(-2.0^{*}\right)$ & $1.63(+2.2)$ & $11.1^{*}$ \\
\hline
\end{tabular}

\begin{tabular}{|c|c|c|c|c|c|c|c|}
\hline & \multicolumn{7}{|c|}{ value } \\
\hline Case & $m_{1} \cdot 10^{2} \mathrm{eV}^{-2}$ & $m_{2} \cdot 10^{2} \mathrm{eV}^{-2}$ & $m_{3} \cdot 10^{2} \mathrm{eV}^{-2}$ & $\alpha_{21} / \pi$ & $\alpha_{31} / \pi$ & $m_{e e} \cdot 10^{2} \mathrm{eV}^{-1}$ & MO \\
\hline $5 W C 3$ & 4.94 & 5.01 & 0.0942 & 0.70 & 0.94 & 2.7 & IO \\
\hline $5 W C 3 p$ & 2.82 & 2.95 & 5.76 & 0.38 & 0.26 & 2.3 & NO \\
\hline Case & $m_{1} \cdot 10^{2} \mathrm{eV}^{-2}$ & $m_{2} \cdot 10^{2} \mathrm{eV}^{-2}$ & $m_{3} \cdot 10^{2} \mathrm{eV}^{-2}$ & $\left(\alpha_{21}-\alpha_{31}\right) / \pi$ & $m_{e e} \cdot 10^{2} \mathrm{eV}^{-1}$ & $\mathrm{MO}$ \\
\hline $5 S C$ & 0 & 0.860 & 5.02 & \multicolumn{2}{|c|}{1.68} & 0.13 & NO \\
\hline
\end{tabular}

\begin{tabular}{|c|c|c|c|c|c|c|c|c|c|c|}
\hline & \multicolumn{10}{|c|}{ Input parameters } \\
\hline Case & $\operatorname{Re}(\tau)$ & $\operatorname{Im}(\tau)$ & $\operatorname{Re}\left(\varphi_{2}\right)$ & $\operatorname{Im}\left(\varphi_{2}\right)$ & $\operatorname{Re}\left(\varphi_{3}\right)$ & $\operatorname{Im}\left(\varphi_{3}\right)$ & $\alpha \cdot 10^{3}$ & $\beta \cdot 10^{3}$ & $\gamma \cdot 10^{3}$ & $1 / \Lambda\left(\mathrm{eV}^{-1}\right)$ \\
\hline $5 W C 3$ & -0.01882 & 0.9929 & 0.4260 & - & 0.8030 & - & 3.018 & 3.927 & -0.4484 & 0.008180 \\
\hline $5 W C 3 p$ & -0.09033 & 0.2190 & 0.4244 & - & 0.01694 & - & 3.259 & 4.311 & -0.8036 & 0.0006303 \\
\hline $5 S C$ & -0.3615 & 0.2412 & 0.04759 & - & 0.3731 & - & 3.368 & 4.411 & -0.8126 & 0.0001639 \\
\hline
\end{tabular}

Table 8. Results of the fit to lepton data for the $A_{5}$ models. For the $5 S C$ case, the predicted lightest neutrino mass is $m_{1}=0$ and so only one physical Majorana phase exists, which appears in the combination $\left(\alpha_{21}-\alpha_{31}\right)$ in neutrinoless double beta decay and hence we report only this combination. We have fixed $\varphi_{1}=1$ for all three cases. ${ }^{*}$ Actual NuFit 4.0 error on $\sin ^{2} \theta_{23}$ measurement (for NO) used, rather than assumed Gaussian error. To simplify the notation, the factors $\cos \hat{\beta}$ and $1 / \sin ^{2} \hat{\beta}$ have been omitted from $\alpha, \beta, \gamma$ and $\Lambda$, respectively.

\section{Conclusion}

Modular invariance have been proven to offer a promising framework to describe lepton masses and mixing angles. In minimal models masses, mixing angles and phases are all predicted in terms of the modulus in addition to a few free parameters. Despite these nice features, neutrinos and charged leptons typically require different realizations to reproduce the sizeable hierarchy among electron, muon and tau masses. In most of the existing models right-handed leptons are assign to singlets of the modular group to allow a sufficient number of free parameters, tuned to match the charged lepton masses. We think that this aspect might indicate the need for a different description, perhaps in terms of other moduli than the one controlling the neutrino sector. In a simple-minded approach, not aiming at a fundamental description but rather to test the ground for a more extensive analysis, we have explored alternative realisations of the charged lepton sector in modular invariant models at levels 4 and 5 .

At level 4 we have shown that it is possible to ascribe the charged lepton mass hierarchy to the weight difference in the right handed sector, similar to what occurs in Froggatt-Nielsen models, wherein the role of the weights is played by the charges. At level 5 we have assigned both right-handed and left-handed leptons to irreducible triplets of the finite modular group $\Gamma_{5}$. Moreover we have shown that also at level 5 the three param- 
eters required to describe charged lepton masses can be almost within the same order of magnitude, though requiring some degree of tuning. In all models considered here we do not need a strong hierarchy at the level of Lagrangian parameters to reproduce charged lepton masses.

We built several models along these lines, analysing neutrino masses coming either from the Weinberg operator or from a type I seesaw, and we have selected seven scenarios which produce a reasonable fit to data, four of them at level 4 and three at level 5 . We looked for minimal realisations, in terms of the lowest possible number of free parameters. Among them we also count the vacuum expectation values of both modulus and flavons, which we varied in order to maximise the agreement with the data. Three parameters are in a one-to-one relation with the charged lepton masses. Besides them, all of our scenarios make use of five parameters, always including an overall scale $\Lambda$, and real and imaginary parts of $\tau$. In these cases we get four predictions: the absolute neutrino mass scale and all $C P$ violating phases, which allow one to pin down the value of $m_{e e}$, relevant to neutrinoless double beta decay. So far few models based on modular invariance perform better, managing to fit the neutrino data with four free parameters. In all cases analysed at level 5 and in two cases at level 4 we demanded that the Lagrangian be $C P$ conserving. A common feature of level 4 and 5 scenarios is that inverted ordering for neutrino masses is predicted when adopting the Weinberg operator and normal ordering when making use of type I seesaw, with a single exception whose $\chi_{\min }^{2}$ is not particularly good. At level 4 the overall results and predictions are quite stable with respect to the details of the model, only depending on the choice between the Weinberg operator and the seesaw mechanism. In both cases the neutrino mass spectrum is nearly degenerate and the lightest neutrino mass is around $40 \mathrm{meV}$. At level 5 we get an excellent $\chi_{\min }^{2}$ only when considering neutrino masses generated by the Weinberg operator, predicting inverted mass ordering. In the seesaw scenario a good fit requires the introduction of additional parameters. Remarkably we find that our seesaw models at level 5 predict a massless neutrino.

A weak point of our construction is the correct vacuum selection. We have not attempted to dynamically select the vacuum configurations in the symmetry breaking sector, while our results, relying on ordinary flavons besides the modular parameter, require a specific vacuum alignment. We have treated the VEVs as free parameters, to be varied to match the experimental data. The VEV pattern suggested by data is peculiar and points to a nontrivial vacuum selection mechanism, where such an elucidation goes beyond the scope of this paper. We do not consider our results conclusive and we think that there is still a considerable room to improve the characterization of the charged lepton sector. Nevertheless, by exploring some nonstandard possibilities, we hope to have provided some new element for the identification of a basic framework.

\section{Acknowledgments}

This project has received support in part by the MIUR-PRIN project 2015P5SBHT 003 "Search for the Fundamental Laws and Constituents" and by the European Union's Horizon 2020 research and innovation programme under the Marie Sklodowska-Curie grant agree- 
ment $\mathrm{N}^{\circ} 674896$ and 690575 . The research of F. F. was supported in part by the INFN. The research of J. C. C. was supported by the Spanish MINECO project FPA2016-78220-C31-P, the Junta de Andalucía grant FQM101 and the Spanish MECD grant FPU14. SJDK would like to thank Gui-Jun Ding for useful discussions.

\section{A Finite modular group $\Gamma_{4}$ and level 4 modular forms}

The finite modular group $\Gamma_{4}$ is isomorphic to $S_{4}$, the symmetric group of permutations of four objects. It has 24 elements and five irreducible representations: $\mathbf{1}, \mathbf{1}^{\prime}, \mathbf{2}, \mathbf{3}$ and $\mathbf{3}^{\prime}$. It admits a presentation in terms of two generators $S$ and $T$ :

$$
S^{2}=(S T)^{3}=T^{4}=\mathbb{1} .
$$

In this paper we use an explicit realization of the elements $S$ and $T$ for the different representations, obtained from the one in ref. [61], with the identification [48]: $S=S^{\prime} T^{2}$ and $T=S^{\prime}$, where the primed generators are those given in ref. [61]. We also use the Clebsch-Gordan coefficients listed in ref. [61].

The linear space of weight 2 and level 4 modular forms has dimension 5 (see, e.g., [10]). These forms can be constructed in terms of the Dedekind eta function [48]:

$$
\eta(\tau) \equiv q^{1 / 24} \prod_{n=1}^{\infty}\left(1-q^{n}\right), \quad q=e^{2 \pi i \tau} .
$$

Defining

$$
\begin{aligned}
Y\left(c_{1}, \ldots, c_{6} \mid \tau\right) \equiv \frac{\mathrm{d}}{\mathrm{d} \tau}[ & c_{1} \log \eta\left(\tau+\frac{1}{2}\right)+c_{2} \log \eta(4 \tau)+c_{3} \log \eta\left(\frac{\tau}{4}\right) \\
& \left.+c_{4} \log \eta\left(\frac{\tau+1}{4}\right)+c_{5} \log \eta\left(\frac{\tau+2}{4}\right)+c_{6} \log \eta\left(\frac{\tau+3}{4}\right)\right],
\end{aligned}
$$

with $c_{1}+\cdots+c_{6}=0$, the basis of the modular forms of weight 2 reads [48],

$$
\begin{aligned}
& Y_{1}(\tau) \equiv i Y\left(1,1, \omega, \omega^{2}, \omega, \omega^{2} \mid \tau\right) \\
& Y_{2}(\tau) \equiv i Y\left(1,1, \omega^{2}, \omega, \omega^{2}, \omega \mid \tau\right) \\
& Y_{3}(\tau) \equiv i Y(1,-1,-1,-1,1,1 \mid \tau) \\
& Y_{4}(\tau) \equiv i Y\left(1,-1,-\omega^{2},-\omega, \omega^{2}, \omega \mid \tau\right) \\
& Y_{5}(\tau) \equiv i Y\left(1,-1,-\omega,-\omega^{2}, \omega, \omega^{2} \mid \tau\right)
\end{aligned}
$$

with $\omega \equiv e^{2 \pi i / 3}$. Notice here, we have an extra factor of $i$ compared to the definition of ref. [48]. It has been shown that $Y_{1}(\tau)$ and $Y_{2}(\tau)$ form a doublet transforming in the $\mathbf{2}$ of $S_{4}$, while the three remaining modular forms make up a triplet transforming in $\mathbf{3}^{\prime}$ of $S_{4}$. Doublet and the triplet will be denoted by

$$
Y_{\mathbf{2}}(\tau) \equiv\left(\begin{array}{c}
Y_{1}(\tau) \\
Y_{2}(\tau)
\end{array}\right), \quad Y_{\mathbf{3}^{\prime}}(\tau) \equiv\left(\begin{array}{c}
Y_{3}(\tau) \\
Y_{4}(\tau) \\
Y_{5}(\tau)
\end{array}\right)
$$


The $q$-expansions $\left(q \equiv e^{i 2 \pi \tau}\right)$ for eq. (A.4) can be found in [48]. In our analysis we use the full analytic form. The modular forms of higher weights $k=4,6, \ldots$ are homogeneous polynomials in the variables $Y_{i}(\tau), i=1, \ldots, 5$.

Under $C P$, eq. (2.9), modular forms of level 4 and weight 2 transform as [49]:

$$
Y_{\mathbf{2}}\left(-\tau^{*}\right)=X_{\mathbf{2}}\left[Y_{\mathbf{2}}(\tau)\right]^{*}, \quad Y_{\mathbf{3}^{\prime}}\left(-\tau^{*}\right)=X_{\mathbf{3}^{\prime}}\left[Y_{\mathbf{3}^{\prime}}(\tau)\right]^{*}
$$

where $X_{\mathbf{2}}$ and $X_{\mathbf{3}^{\prime}}$ are the matrices:

$$
X_{\mathbf{2}}=\left(\begin{array}{ll}
0 & 1 \\
1 & 0
\end{array}\right) \quad, \quad X_{\mathbf{3}^{\prime}}=-\frac{1}{3}\left(\begin{array}{ccc}
-1 & 2 \omega & 2 \omega^{2} \\
2 \omega & 2 \omega^{2} & -1 \\
2 \omega^{2} & -1 & 2 \omega
\end{array}\right) .
$$

By decomposing products of representations in their irreducible components we find that a consistent action of $C P$ on chiral multiplets $\varphi_{\mathbf{r}}$ transforming in the representation $\mathbf{r}$ $\left(\mathbf{r}=\mathbf{1}, \mathbf{1}^{\prime}, \mathbf{2}, \mathbf{3}, \mathbf{3}^{\prime}\right)$ of $\Gamma_{4}$ is given by:

$$
\varphi_{\mathbf{r}} \stackrel{C P}{\longrightarrow} X_{\mathbf{r}} \varphi_{\mathbf{r}}^{*}
$$

with $X_{2}$ and $X_{3^{\prime}}$ given above and

$$
X_{1}=-X_{1^{\prime}}=1 \quad, \quad X_{3}=X_{3^{\prime}}
$$

This set of matrices satisfy the consistency conditions

$$
X_{\mathbf{r}} \rho_{\mathbf{r}}^{*}(\gamma) X_{\mathbf{r}}^{-1}=\rho_{\mathbf{r}}\left(\gamma^{\prime}\right), \quad\left(\gamma, \gamma^{\prime}\right) \in \Gamma,
$$

as can be checked by working with the generators $\gamma=(S, T)$. We find $S^{\prime}=S^{-1}$ and $T^{\prime}=T^{-1}$. The conditions for the $\operatorname{VEV}\left(\varphi_{1}, \varphi_{2}, \varphi_{3}\right)$ of a triplet to preserve $C P$ read:

$$
\operatorname{Im}\left(\varphi_{1}\right)=\sqrt{3} \operatorname{Re}\left(\varphi_{2}-\varphi_{3}\right), \quad \operatorname{Im}\left(\varphi_{2}\right)=\sqrt{3} \operatorname{Re}\left(\varphi_{1}-\varphi_{2}\right), \quad \operatorname{Im}\left(\varphi_{3}\right)=\sqrt{3} \operatorname{Re}\left(\varphi_{3}-\varphi_{1}\right)
$$

In our basis, the requirement of $C P$ conservation on a modular invariant supersymmetric theory at level 4 , adopting the above $C P$ transformations on the chiral multiplets, amounts to having all Lagrangian parameters real.

\section{B Finite modular group $\Gamma_{5}$ and level 5 modular forms}

The finite modular group $\Gamma_{5}$ is isomorphic to $A_{5}$, the group of even permutations of five objects. It has 60 elements and five irreducible representations: $\mathbf{1}, \mathbf{3}, \mathbf{3}^{\prime}, \mathbf{4}$ and $\mathbf{5}$. It admits a presentation in terms of two generators of $S$ and $T$ :

$$
S^{2}=(S T)^{3}=T^{5}=I .
$$

In this paper we use the explicit realisation of the elements $S$ and $T$ for the different representations given in ref. [62], where we can also find the corresponding Clebsch-Gordan coefficients. 
Level 5 modular forms of weight 2 have been built in ref. [51], making use of the Jacobi theta function:

$$
\theta_{3}(u, \tau) \equiv \theta_{0,0}(u, \tau)=\sum_{n=-\infty}^{\infty} p^{n^{2}} e^{2 \pi i n u}
$$

where $p \equiv e^{\pi i \tau}$. Defining the seed functions:

$$
\begin{aligned}
\alpha_{1,-1}(\tau) & \equiv \theta_{3}\left(\frac{\tau+1}{2}, 5 \tau\right), & \alpha_{2,-1}(\tau) & \equiv e^{2 \pi i \tau / 5} \theta_{3}\left(\frac{3 \tau+1}{2}, 5 \tau\right), \\
\alpha_{1,0}(\tau) & \equiv \theta_{3}\left(\frac{\tau+9}{10}, \frac{\tau}{5}\right), & \alpha_{2,0}(\tau) & \equiv \theta_{3}\left(\frac{\tau+7}{10}, \frac{\tau}{5}\right) \\
\alpha_{1,1}(\tau) & \equiv \theta_{3}\left(\frac{\tau}{10}, \frac{\tau+1}{5}\right), & \alpha_{2,1}(\tau) & \equiv \theta_{3}\left(\frac{\tau+8}{10}, \frac{\tau+1}{5}\right) \\
\alpha_{1,2}(\tau) & \equiv \theta_{3}\left(\frac{\tau+1}{10}, \frac{\tau+2}{5}\right), & \alpha_{2,2}(\tau) & \equiv \theta_{3}\left(\frac{\tau+9}{10}, \frac{\tau+2}{5}\right) \\
\alpha_{1,3}(\tau) & \equiv \theta_{3}\left(\frac{\tau+2}{10}, \frac{\tau+3}{5}\right), & \alpha_{2,3}(\tau) & \equiv \theta_{3}\left(\frac{\tau}{10}, \frac{\tau+3}{5}\right) \\
\alpha_{1,4}(\tau) & \equiv \theta_{3}\left(\frac{\tau+3}{10}, \frac{\tau+4}{5}\right), & \alpha_{2,4}(\tau) & \equiv \theta_{3}\left(\frac{\tau+1}{10}, \frac{\tau+4}{5}\right)
\end{aligned}
$$

and the functions,

$$
Y\left(c_{1,-1}, \ldots, c_{1,4} ; c_{2,-1}, \ldots, c_{2,4} \mid \tau\right) \equiv \sum_{i, j} c_{i, j} \frac{\mathrm{d}}{\mathrm{d} \tau} \log \alpha_{i, j}(\tau), \quad \text { with } \sum_{i, j} c_{i, j}=0
$$

then the modular forms of weight two are divided into the following multiplets of $A_{5}$,

$$
\begin{gathered}
Y_{\mathbf{5}}(\tau)=\left(\begin{array}{l}
Y_{1}(\tau) \\
Y_{2}(\tau) \\
Y_{3}(\tau) \\
Y_{4}(\tau) \\
Y_{5}(\tau)
\end{array}\right) \equiv\left(\begin{array}{c}
-\frac{1}{\sqrt{6}} Y(-5,1,1,1,1,1 ;-5,1,1,1,1,1 \mid \tau) \\
Y\left(0,1, \zeta^{4}, \zeta^{3}, \zeta^{2}, \zeta ; 0,1, \zeta^{4}, \zeta^{3}, \zeta^{2}, \zeta \mid \tau\right) \\
Y\left(0,1, \zeta^{3}, \zeta, \zeta^{4}, \zeta^{2} ; 0,1, \zeta^{3}, \zeta, \zeta^{4}, \zeta^{2} \mid \tau\right) \\
Y\left(0,1, \zeta^{2}, \zeta^{4}, \zeta, \zeta^{3} ; 0,1, \zeta^{2}, \zeta^{4}, \zeta, \zeta^{3} \mid \tau\right) \\
Y\left(0,1, \zeta, \zeta^{2}, \zeta^{3}, \zeta^{4} ; 0,1, \zeta, \zeta^{2}, \zeta^{3}, \zeta^{4} \mid \tau\right)
\end{array}\right), \\
Y_{\mathbf{3}}(\tau)=\left(\begin{array}{c}
Y_{6}(\tau) \\
Y_{7}(\tau) \\
Y_{8}(\tau)
\end{array}\right) \equiv\left(\begin{array}{c}
\frac{1}{\sqrt{2}} Y(-\sqrt{5},-1,-1,-1,-1,-1 ; \sqrt{5}, 1,1,1,1,1 \mid \tau) \\
Y\left(0,1, \zeta^{4}, \zeta^{3}, \zeta^{2}, \zeta ; 0,-1,-\zeta^{4},-\zeta^{3},-\zeta^{2},-\zeta \mid \tau\right) \\
Y\left(0,1, \zeta, \zeta^{2}, \zeta^{3}, \zeta^{4} ; 0,-1,-\zeta,-\zeta^{2},-\zeta^{3},-\zeta^{4} \mid \tau\right)
\end{array}\right), \\
Y_{3^{\prime}}(\tau)=\left(\begin{array}{c}
Y_{9}(\tau) \\
Y_{10}(\tau) \\
Y_{11}(\tau)
\end{array}\right) \equiv\left(\begin{array}{c}
\frac{1}{\sqrt{2}} Y(\sqrt{5},-1,-1,-1,-1,-1 ;-\sqrt{5}, 1,1,1,1,1 \mid \tau) \\
Y\left(0,1, \zeta^{3}, \zeta, \zeta^{4}, \zeta^{2} ; 0,-1,-\zeta^{3},-\zeta,-\zeta^{4},-\zeta^{2} \mid \tau\right) \\
Y\left(0,1, \zeta^{2}, \zeta^{4}, \zeta, \zeta^{3} ; 0,-1,-\zeta^{2},-\zeta^{4},-\zeta,-\zeta^{3} \mid \tau\right)
\end{array}\right),
\end{gathered}
$$

where $\zeta=e^{2 \pi i / 5}$. The first few terms of the $q$-expansions of these modular forms can be found in ref. [51]. Our numerical results have made use of $q$-expansions up to $\mathcal{O}\left(q^{100}\right)$, but the results are unchanged when using up to $\mathcal{O}\left(q^{5}\right)$. 


\begin{tabular}{|c|c|c|c|c|c|c|c|c|c|c|}
\hline & \multicolumn{10}{|c|}{ Input parameters --- fundamental region } \\
\hline Case & $\gamma \tau$ & $\operatorname{Re}(\tau)$ & $\operatorname{Im}(\tau)$ & $\operatorname{Re}\left(\varphi_{1}\right)$ & $\operatorname{Im}\left(\varphi_{1}\right)$ & $\operatorname{Re}\left(\varphi_{2}\right)$ & $\operatorname{Im}\left(\varphi_{2}\right)$ & $\operatorname{Re}\left(\varphi_{3}\right)$ & $\operatorname{Im}\left(\varphi_{3}\right)$ & $1 / \Lambda\left(\mathrm{eV}^{-1}\right)$ \\
\hline $4 W V$ & $S T^{-1} \tau$ & -0.1579 & 0.9957 & $2 / 3$ & 0 & $1 / 6$ & $1 / 2 \sqrt{3}$ & $-1 / 3$ & $1 / \sqrt{3}$ & 0.003223 \\
\hline $4 S V$ & $T^{-1} \tau$ & -0.1564 & 0.9968 & $-1 / 3$ & $-1 / \sqrt{3}$ & $-1 / 3$ & $1 / \sqrt{3}$ & $-1 / 3$ & 0 & 0.7672 \\
\hline $4 W C$ & $T^{-1} S T^{-3} \tau$ & -0.07915 & 1.055 & -0.3947 & 0.5774 & 0.6974 & -0.05315 & 0.1053 & 0.1824 & 0.0007030 \\
\hline $4 S C$ & $T^{-1} S T^{-3} \tau$ & -0.1667 & 0.9966 & -0.2709 & 0.5774 & 0.6355 & 0.05406 & 0.2291 & 0.3968 & 0.06993 \\
\hline
\end{tabular}

Table 9. Parameters $\tau$ and $\varphi$ in the fundamental region for level 4 models.

\begin{tabular}{|c|c|c|c|c|c|c|c|c|c|c|}
\hline & \multicolumn{10}{|c|}{ Input parameters --- fundamental region } \\
\hline Case & $\gamma \tau$ & $\operatorname{Re}(\tau)$ & $\operatorname{Im}(\tau)$ & $\operatorname{Re}\left(\varphi_{1}\right)$ & $\operatorname{Im}\left(\varphi_{1}\right)$ & $\operatorname{Re}\left(\varphi_{2}\right)$ & $\operatorname{Im}\left(\varphi_{2}\right)$ & $\operatorname{Re}\left(\varphi_{3}\right)$ & $\operatorname{Im}\left(\varphi_{3}\right)$ & $1 / \Lambda\left(\mathrm{eV}^{-1}\right)$ \\
\hline $5 W C 3$ & $S \tau$ & 0.01908 & 1.007 & -0.3301 & 0 & -0.7188 & 0 & -1.096 & 0 & 0.007958 \\
\hline $5 W C 3 p$ & $T^{-2} S \tau$ & -0.3908 & 3.902 & -0.1618 & 0 & 0.1621 & 0.4990 & 0.2911 & -0.8960 & 0.0007302 \\
\hline $5 S C$ & $T^{-2} S \tau$ & -0.08591 & 1.277 & 0.1812 & 0 & 0.4561 & 0.3314 & 0.7194 & -0.5227 & 0.002804 \\
\hline
\end{tabular}

Table 10. Parameters $\tau$ and $\varphi$ in the fundamental region for level 5 models.

\section{Numerical results in fundamental region}

The models studied in this paper are modular invariant and it is always possible to map the Lagrangian referred to a certain value $\tau$ of the modulus to an equivalent Lagrangian where the modulus $\tau^{\prime}$ is inside the fundamental region $\left|\operatorname{Re}\left(\tau^{\prime}\right)\right| \leq 1 / 2,\left|\tau^{\prime}\right| \geq 1$. By definition there exists a modular transformation $\gamma$ such that $\tau^{\prime}=\gamma \tau$. Together with the transformation $\tau \rightarrow \gamma \tau$, we consider the field redefinition mapping all chiral multiplets except $L$ into the modular transformed ones, after setting to zero all their weights. We find that the low-energy superpotential

$$
w=-\frac{v^{2}}{2 \Lambda} L^{T} \mathcal{W}(\tau) L-\frac{v}{\sqrt{2}} E^{c T} \mathcal{Y}(\varphi) L
$$

becomes

$$
w=-\frac{v^{2}}{2 \Lambda} L^{T} \mathcal{W}(\gamma \tau) L-\frac{v}{\sqrt{2}} E^{c T} \mathcal{Y}(\varphi) \rho_{L}^{\dagger}(\gamma) L
$$

where

$$
\mathcal{W}(\gamma \tau)=(c \tau+d)^{2} \rho_{L}(\gamma)^{*} \mathcal{W}(\tau) \rho_{L}^{\dagger}(\gamma)
$$

Neutrino and charged lepton mass matrices are now:

$$
m_{\nu}=\frac{v^{2}}{\Lambda} \mathcal{W}(\gamma \tau), \quad m_{e}^{\dagger} m_{e}=\frac{v^{2}}{2} \rho_{L}(\gamma) \mathcal{Y}(\varphi)^{\dagger} \mathcal{Y}(\varphi) \rho_{L}^{\dagger}(\gamma)
$$

The lepton mixing matrix is unchanged. We list here the transformations needed to map the values of $\tau$ found by our minimisation procedure to points inside the fundamental region.

Open Access. This article is distributed under the terms of the Creative Commons Attribution License (CC-BY 4.0), which permits any use, distribution and reproduction in any medium, provided the original author(s) and source are credited. 


\section{References}

[1] Y. Reyimuaji and A. Romanino, Can an unbroken flavour symmetry provide an approximate description of lepton masses and mixing?, JHEP 03 (2018) 067 [arXiv:1801.10530] [INSPIRE].

[2] G. Altarelli and F. Feruglio, Discrete flavor symmetries and models of neutrino mixing, Rev. Mod. Phys. 82 (2010) 2701 [arXiv: 1002.0211] [InSPIRE].

[3] H. Ishimori et al., Non-Abelian discrete symmetries in particle physics, Prog. Theor. Phys. Suppl. 183 (2010) 1 [arXiv:1003.3552] [INSPIRE].

[4] D. Hernandez and A.Yu. Smirnov, Lepton mixing and discrete symmetries, Phys. Rev. D 86 (2012) 053014 [arXiv: 1204.0445] [INSPIRE].

[5] S.F. King and C. Luhn, Neutrino mass and mixing with discrete symmetry, Rept. Prog. Phys. 76 (2013) 056201 [arXiv: 1301.1340] [INSPIRE].

[6] S.F. King et al., Neutrino mass and mixing: from theory to experiment, New J. Phys. 16 (2014) 045018 [arXiv: 1402.4271] [INSPIRE].

[7] F. Feruglio, Pieces of the flavour puzzle, Eur. Phys. J. C 75 (2015) 373 [arXiv:1503.04071] [INSPIRE].

[8] S.F. King, Unified models of neutrinos, flavour and CP-violation, Prog. Part. Nucl. Phys. 94 (2017) 217 [arXiv: 1701.04413] [INSPIRE].

[9] C. Hagedorn, Theories of leptonic flavor, arXiv:1705.00684 [INSPIRE].

[10] F. Feruglio, Are neutrino masses modular forms?, arXiv:1706.08749 [INSPIRE].

[11] P.P. Novichkov, J.T. Penedo, S.T. Petcov and A.V. Titov, Generalised CP symmetry in modular-invariant models of flavour, JHEP 07 (2019) 165 [arXiv: 1905.11970] [INSPIRE].

[12] T. Dent, CP violation and modular symmetries, Phys. Rev. D 64 (2001) 056005 [hep-ph/0105285] [INSPIRE].

[13] T. Dent, On the modular invariance of mass eigenstates and CP-violation, JHEP 12 (2001) 028 [hep-th/0111024] [INSPIRE].

[14] A. Baur, H.P. Nilles, A. Trautner and P.K.S. Vaudrevange, Unification of flavor, CP and modular symmetries, Phys. Lett. B 795 (2019) 7 [arXiv:1901.03251] [INSPIRE].

[15] A. Baur, H.P. Nilles, A. Trautner and P.K.S. Vaudrevange, A string theory of flavor and CP, Nucl. Phys. B 947 (2019) 114737 [arXiv:1908.00805] [InSPIRE].

[16] S. Ferrara, D. Lüst and S. Theisen, Target Space modular invariance and low-energy couplings in orbifold compactifications, Phys. Lett. B 233 (1989) 147 [INSPIRE].

[17] I. De Medeiros Varzielas, S.F. King and Y.-L. Zhou, Multiple modular symmetries as the origin of flavour, arXiv:1906.02208 [INSPIRE].

[18] S. Hamidi and C. Vafa, Interactions on orbifolds, Nucl. Phys. B 279 (1987) 465 [inSPIRE].

[19] L.J. Dixon, D. Friedan, E.J. Martinec and S.H. Shenker, The conformal field theory of orbifolds, Nucl. Phys. B 282 (1987) 13 [INSPIRE].

[20] J. Lauer, J. Mas and H.P. Nilles, Duality and the role of nonperturbative effects on the world sheet, Phys. Lett. B 226 (1989) 251 [inSPIRE]. 
[21] J. Lauer, J. Mas and H.P. Nilles, Twisted sector representations of discrete background symmetries for two-dimensional orbifolds, Nucl. Phys. B 351 (1991) 353 [INSPIRE].

[22] J. Erler, D. Jungnickel and J. Lauer, Dependence of Yukawa couplings on the axionic background moduli of $Z(N)$ orbifolds, Phys. Rev. D 45 (1992) 3651 [INSPIRE].

[23] D. Cremades, L.E. Ibáñez and F. Marchesano, Yukawa couplings in intersecting D-brane models, JHEP 07 (2003) 038 [hep-th/0302105] [INSPIRE].

[24] R. Blumenhagen, M. Cvetič, P. Langacker and G. Shiu, Toward realistic intersecting D-brane models, Ann. Rev. Nucl. Part. Sci. 55 (2005) 71 [hep-th/0502005] [InSPIRE].

[25] S.A. Abel and M.D. Goodsell, Realistic Yukawa couplings through instantons in intersecting brane worlds, JHEP 10 (2007) 034 [hep-th/0612110] [INSPIRE].

[26] R. Blumenhagen, B. Körs, D. Lüst and S. Stieberger, Four-dimensional string compactifications with D-branes, orientifolds and fluxes, Phys. Rept. 445 (2007) 1 [hep-th/0610327] [INSPIRE].

[27] F. Marchesano, Progress in D-brane model building, Fortsch. Phys. 55 (2007) 491 [hep-th/0702094] [INSPIRE].

[28] I. Antoniadis, A. Kumar and B. Panda, Fermion wavefunctions in magnetized branes: theta identities and Yukawa couplings, Nucl. Phys. B 823 (2009) 116 [arXiv:0904.0910] [INSPIRE].

[29] T. Kobayashi, S. Nagamoto and S. Uemura, Modular symmetry in magnetized/intersecting D-brane models, PTEP 2017 (2017) 023B02 [arXiv: 1608.06129] [INSPIRE].

[30] D. Cremades, L.E. Ibáñez and F. Marchesano, Computing Yukawa couplings from magnetized extra dimensions, JHEP 05 (2004) 079 [hep-th/0404229] [INSPIRE].

[31] H. Abe, K.-S. Choi, T. Kobayashi and H. Ohki, Non-abelian discrete flavor symmetries from magnetized/intersecting brane models, Nucl. Phys. B 820 (2009) 317 [arXiv:0904.2631] [INSPIRE].

[32] T. Kobayashi et al., Modular symmetry and non-Abelian discrete flavor symmetries in string compactification, Phys. Rev. D 97 (2018) 116002 [arXiv:1804.06644] [INSPIRE].

[33] L.E. Ibáñez, Hierarchy of quark-lepton masses in orbifold superstring compactification, Phys. Lett. B 181 (1986) 269 [INSPIRE].

[34] J.A. Casas, F. Gómez and C. Muñoz, Complete structure of Z(n) Yukawa couplings, Int. J. Mod. Phys. A 8 (1993) 455 [hep-th/9110060] [INSPIRE].

[35] O. Lebedev, The CKM phase in heterotic orbifold models, Phys. Lett. B 521 (2001) 71 [hep-th/0108218] [INSPIRE].

[36] T. Kobayashi and O. Lebedev, Heterotic Yukawa couplings and continuous Wilson lines, Phys. Lett. B 566 (2003) 164 [hep-th/0303009] [INSPIRE].

[37] P. Brax and M. Chemtob, Flavor changing neutral current constraints on standard-like orbifold models, Phys. Rev. D 51 (1995) 6550 [hep-th/9411022] [INSPIRE].

[38] P. Binetruy and E. Dudas, Dynamical mass matrices from effective superstring theories, Nucl. Phys. B 451 (1995) 31 [hep-ph/9505295] [INSPIRE].

[39] E. Dudas, S. Pokorski and C.A. Savoy, Soft scalar masses in supergravity with horizontal U(1) - x gauge symmetry, Phys. Lett. B 369 (1996) 255 [hep-ph/9509410] [InSPIRE]. 
[40] E. Dudas, Dynamical mass matrices from moduli fields, hep-ph/9602231 [INSPIRE].

[41] G.K. Leontaris and N.D. Tracas, Modular weights, U(1)'s and mass matrices, Phys. Lett. B 419 (1998) 206 [hep-ph/9709510] [INSPIRE].

[42] T. Kobayashi, K. Tanaka and T.H. Tatsuishi, Neutrino mixing from finite modular groups, Phys. Rev. D 98 (2018) 016004 [arXiv:1803.10391] [INSPIRE].

[43] T. Kobayashi et al., Finite modular subgroups for fermion mass matrices and baryon/lepton number violation, Phys. Lett. B 794 (2019) 114 [arXiv:1812.11072] [INSPIRE].

[44] J.C. Criado and F. Feruglio, Modular invariance faces precision neutrino data, SciPost Phys. 5 (2018) 042 [arXiv: 1807.01125] [INSPIRE].

[45] T. Kobayashi et al., Modular $A_{4}$ invariance and neutrino mixing, JHEP 11 (2018) 196 [arXiv: 1808.03012] [INSPIRE].

[46] P.P. Novichkov, S.T. Petcov and M. Tanimoto, Trimaximal neutrino mixing from modular $A_{4}$ invariance with residual symmetries, Phys. Lett. B 793 (2019) 247 [arXiv:1812.11289] [INSPIRE].

[47] G.-J. Ding, S.F. King and X.-G. Liu, Modular $A_{4}$ symmetry models of neutrinos and charged leptons, JHEP 09 (2019) 074 [arXiv: 1907.11714] [INSPIRE].

[48] J.T. Penedo and S.T. Petcov, Lepton masses and mixing from modular $S_{4}$ symmetry, Nucl. Phys. B 939 (2019) 292 [arXiv: 1806.11040] [INSPIRE].

[49] P.P. Novichkov, J.T. Penedo, S.T. Petcov and A.V. Titov, Modular $S_{4}$ models of lepton masses and mixing, JHEP 04 (2019) 005 [arXiv:1811.04933] [INSPIRE].

[50] S.F. King and Y.-L. Zhou, Trimaximal TM 1 mixing with two modular $S_{4}$ groups, Phys. Rev. D 101 (2020) 015001 [arXiv: 1908.02770] [INSPIRE].

[51] P.P. Novichkov, J.T. Penedo, S.T. Petcov and A.V. Titov, Modular $A_{5}$ symmetry for flavour model building, JHEP 04 (2019) 174 [arXiv: 1812.02158] [INSPIRE].

[52] G.-J. Ding, S.F. King and X.-G. Liu, Neutrino mass and mixing with $A_{5}$ modular symmetry, Phys. Rev. D 100 (2019) 115005 [arXiv:1903.12588] [INSPIRE].

[53] H. Okada and M. Tanimoto, CP violation of quarks in $A_{4}$ modular invariance, Phys. Lett. $\mathbf{B}$ 791 (2019) 54 [arXiv:1812.09677] [INSPIRE].

[54] H. Okada and M. Tanimoto, Towards unification of quark and lepton flavors in $A_{4}$ modular invariance, arXiv:1905.13421 [INSPIRE].

[55] F.J. de Anda, S.F. King and E. Perdomo, SU(5) grand unified theory with $A_{4}$ modular symmetry, arXiv: 1812.05620 [INSPIRE].

[56] T. Kobayashi et al., Modular $S_{3}$ invariant flavor model in SU(5) GUT, arXiv:1906.10341 [INSPIRE].

[57] S. Ferrara, D. Lüst, A.D. Shapere and S. Theisen, Modular invariance in supersymmetric field theories, Phys. Lett. B 225 (1989) 363 [INSPIRE].

[58] X.-G. Liu and G.-J. Ding, Neutrino masses and mixing from double covering of finite modular groups, JHEP 08 (2019) 134 [arXiv:1907.01488] [INSPIRE].

[59] S. Antusch and V. Maurer, Running quark and lepton parameters at various scales, JHEP 11 (2013) 115 [arXiv:1306.6879] [INSPIRE]. 
[60] I. Esteban et al., Global analysis of three-flavour neutrino oscillations: synergies and tensions in the determination of $\theta_{23}, \delta_{\mathrm{CP}}$ and the mass ordering, JHEP 01 (2019) 106 [arXiv: 1811.05487] [INSPIRE].

[61] F. Bazzocchi, L. Merlo and S. Morisi, Fermion masses and mixings in a S(4)-based model, Nucl. Phys. B 816 (2009) 204 [arXiv:0901.2086] [InSPIRE].

[62] G.-J. Ding, L.L. Everett and A.J. Stuart, Golden ratio neutrino mixing and $A_{5}$ flavor symmetry, Nucl. Phys. B 857 (2012) 219 [arXiv:1110.1688] [INSPIRE]. 